\title{
SENTIDO Y EFICACIA DE LA “ANOTACIÓN NEGATIVA” EN EL LIBRO DE CLASES
}

\author{
SENSE AND EFFECTIVENESS OF NOTES \\ ON STUDENT MISBEHAVIOUR
}

\author{
Javier F. A. Vega RamíreZ*
}

\section{Resumen}

El siguiente ensayo analiza el sentido y la eficacia de las observaciones que registra el docente en la hoja de vida del estudiante y en particular la observación denominada "anotación negativa", considerando su uso desde la función discursiva (Austin, 1982) y desde la función dentro del dispositivo de control que prima en la institución escolar (Foucault, 2002). Las principales conclusiones permiten dar cuenta que la anotación negativa, escrita por el profesor, no cumple su función discursiva, ya que está sometida a una verificación por parte de un sistema de control superior, con lo que este elemento administrado por el docente lo convierte, al mismo tiempo, en un recurso de control inconsciente dentro del dispositivo de control que es el sistema escolar y la sociedad. Queda para una discusión posterior el análisis de una posible función de la anotación negativa como objeto de expresión del sentir interno del docente.

Palabras clave: Profesor, escuela, formación, observación, control social, estado y educación.

* Javier F. A. Vega Ramírez. Magíster en Educación, Mención Política y Gestión Educacional (UACH), Universidad Austral de Chile, email: javier.vega@uach.cl 


\begin{abstract}
This article analyzes the use of notes teachers record about students' performance, especially those regarding misbehaviour, considering their use from a point of view of their discursive function (Austin, 1982) and from their function part of the control dispositive in the educational institution (Foucault, 2002). The main conclusions show the notes on misbehaviour recorded by the teacher do not fulfill their discursive function since it is subject to verification by a superior control system, with which this element managed by the teachers converts them, at the same time, in an unconscious control resource within the control device the school system and society are. The analysis of a probable function of the notes on misbehaviour as an object of expression of teachers' internal feeling is left for further discussion.
\end{abstract}

Keywords: Teacher, schools, training, note, social control, state and education

\title{
1. Introducción
}

A EDUCACIÓN ES, contemporáneamente, el ámbito que se ha posicionado con mayor presencia como generador de discursos permanentes, dada la multiplicidad de relaciones que se establecen entre sus actores y la declaración, explícita o implícita, de las concepciones que se tiene sobre cada una de sus acciones. En la multiplicidad de opciones, un eje de análisis interesante, aunque escasamente explorado, es el del registro de anotaciones en el libro de clases por parte de los docentes.

Contamos, a la fecha, sólo con un artículo que da cuenta de una revisión al respecto. El estudio, desarrollado por Alarcón (2015), explora los patrones valorativos que se descubren tras las anotaciones negativas de un centro escolar. El corpus de anotaciones lo componen 174 registros múltiples del año 2005 de un establecimiento de la región metropolitana, cuyo análisis se hace desde la Lingüística Sistémico Funcional (LSF) y el Análisis Crítico del Discurso (ACD). Este estudio se considera importante por considerar la anotación negativa como un género en sí y al mismo tiempo como un dispositivo de control, administrado por un solo actor, con consecuencias para la comunidad educativa (2015, p. 290). 
Esta escasez de estudios relacionados nos permite mayor flexibilidad en el desarrollo de perspectivas nuevas sobre cómo abordar el rol de la anotación negativa en el libro de clases, la posición que esta adopta dentro de la regulación de las relaciones en la comunidad escolar (asumiendo su carácter de dispositivo de control) y, lo que será el objeto propio de este ensayo; la disonancia entre el discurso declarado a través de la anotación negativa y el efecto esperado de este discurso. Para esto, en primer lugar, se hará una revisión documental sobre el rol de la anotación negativa dentro de la comunidad escolar de la escuela tradicional chilena, para ello se utilizarán fuentes documentarias emitidas o validadas por el Ministerio de Educación de Chile (MINEDUC), a continuación, se analizará la eficacia del discurso desde la perspectiva de J. Austin y el enunciado como acto de constatación (Austin, 1982) para, finalmente, verificar el rol que se le atribuye a la anotación negativa, en cuanto recurso de control que se enmarca dentro de un dispositivo de control del que forma parte la comunidad escolar (Foucault, 2002).

\section{Anotación negativa y comunidad escolar}

\subsection{La convivencia como factor de aprendizaje}

El conjunto de actores que interviene en el proceso educativo es múltiple. De manera natural, podemos considerar que el aprendizaje, principalmente, es un acto individual (dado que se aprende según intereses); sin embargo, siempre intervienen otros actores que desencadenan el acto del aprender, como es en primer lugar un otro que cumpla la función de compartir, instruir o guiar el aprendizaje (Ascorra y Crespo, 2004). Desde mediados de 1.800 , se considera el aprendizaje como un acto de construcción social, en donde el otro tiene preponderancia en cuanto que potenciador del aprendizaje. Particularmente importante es la visión de Lev Semionovich Vygotsky (1896-1934), quien profundiza en la noción de aprendizaje desde la teoría sociocultural, con la formulación teórica de la Zona de Desarrollo Próximo (ZDP). Vygotsky plantea que el aprendizaje es un proceso que se da siempre 
en la interacción con otro; aprendizaje que, por lo tanto, se transforma al mismo tiempo en un acto personal que social, dado que requiere de otro referente tanto como contraparte como medida del posible potencial a alcanzar. El autor define la ZDP como:

"distancia entre el nivel real de desarrollo, determinado por la capacidad de resolver independientemente un problema, y el nivel de desarrollo potencial, determinado a través de la resolución de un problema bajo la guía de un adulto o en colaboración con otro compañero más capaz" (Vygotsky, 2009, p. 133).

La comprensión del proceso de aprendizaje, por tanto, se amplía hacia un otro referencial. Esta noción va ampliándose a otros sujetos, desde que se analiza la institución escolar como productora de saberes y reproductora de conocimientos (noción psicológica y sociológica). Hoy en día se comprende el aprendizaje como el resultado de un conjunto de relaciones establecidas entre los sujetos vinculados, en donde participa el estudiante, los docentes; pero también los mundos relacionales del docente y del estudiante, ampliando la red a sujetos como el padre o la madre, el entorno social, los compañeros de estudio, la concepción docente, la noción sobre el aprendizaje, entre otros (figura 1).

Al considerar el fenómeno del aprendizaje como un conjunto de relaciones entre diversos actores, tenemos que considerar que estas relaciones deben establecerse en un conjunto de relaciones ideales, para que ninguno de estos factores se transforme en un elemento negativo o contraproducente. 
Figura 1. Actores intervinientes en el aprendizaje

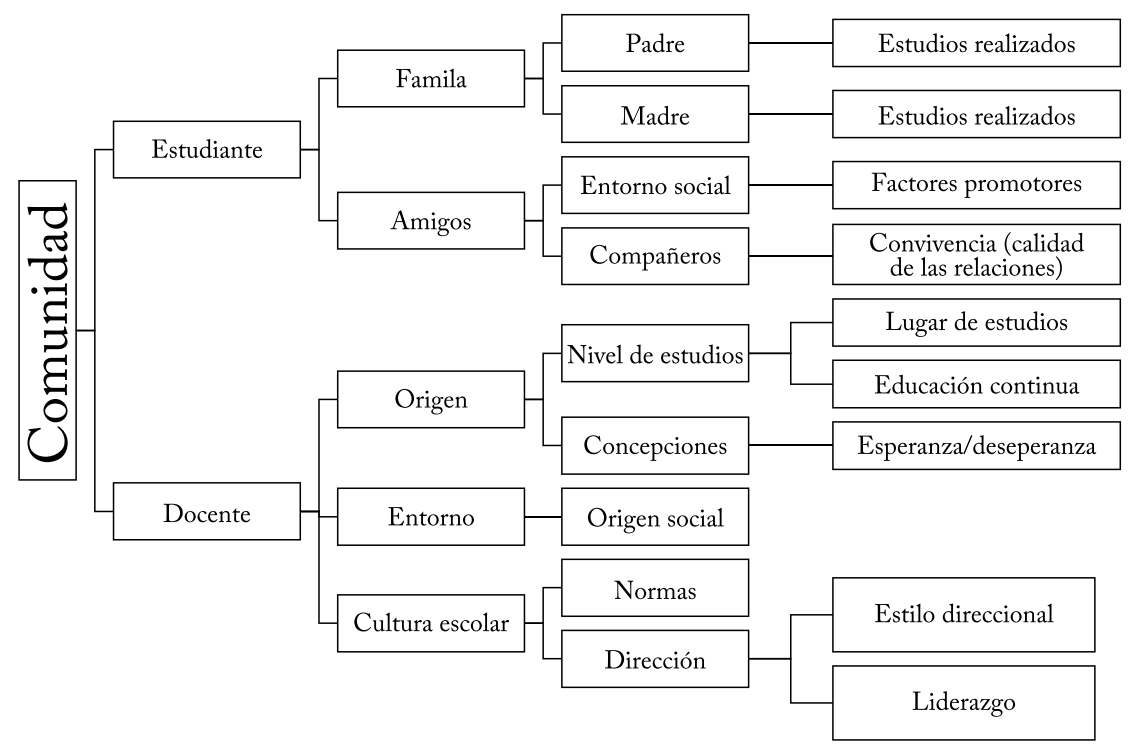

Fuente: Elaboración propia, basada en Brunner y Elaqua (2004); Cornejo y Redondo (2007); Marchesi, Lucena, y Ferrer (2006); Cerón y Lara (2011); Irene y Bareño (2015).

Si bien es difícil establecer con exactitud la influencia de cada uno de los elementos, por ser siempre dinámicos, diversos estudios han intentado establecer su nivel relativo de influencia. Bravo (2008) estableció la relación entre los resultados de aprendizaje y diversos sistemas de evaluación de desempeño de profesores en Chile $\left(\mathrm{AEP}^{1}, \mathrm{AVDI}^{2}\right.$, Evaluación Docente ${ }^{3}$ ), estableciendo diversos niveles de influencia sin que estos necesariamente se tradujeran en efectividad, como estrategia secundaria de apoyo al aprendizaje.

${ }^{1}$ Asignación de Excelencia Pedagógica, sistema de evaluación voluntario de los profesores cuya aplicación estuvo vigente hasta el año 2016, en que es reemplazado por la nueva Carrera Docente.

${ }^{2}$ Asignación Variable de Desempeño Individual, prueba de conocimientos pedagógicos y disciplinarios en uso hasta el año 2016, en que es reemplazado por la nueva Carrera Docente.

${ }^{3}$ Sistema actualmente en uso. 
Por su parte, Vega y Galaz (2015) establecieron, a partir de estos resultados, diversos elementos que tienen relación directa con el aprendizaje, detectando elementos de mayor o menor influencia, basados la mayoría en percepciones, concepciones y sensaciones respecto de la forma en que se aprende (ver Tabla 1).

Tabla 1. Factores intervinientes en el aprendizaje y porcentaje de influencia sobre el rendimiento.

\begin{tabular}{|c|c|c|c|}
\hline \multirow[t]{12}{*}{ Estudiante } & 1. & Nivel Socioeconómico & $9,41 \%$ \\
\hline & 2. & Género & $8,24 \%$ \\
\hline & 3. & Percepción de la disciplina en clases & $1,18 \%$ \\
\hline & 4. & $\begin{array}{l}\text { Preocupación del docente por alumnos con bajo } \\
\text { rendimiento. }\end{array}$ & $4,71 \%$ \\
\hline & 5. & Autoeficacia académica general del estudiante & $8,24 \%$ \\
\hline & 6. & $\begin{array}{l}\text { Motivación por el aprendizaje en Educación } \\
\text { Matemática }\end{array}$ & $7,06 \%$ \\
\hline & 7. & $\begin{array}{l}\text { Autoeficacia académica del estudiante en } \\
\text { Educación Matemática }\end{array}$ & $12,94 \%$ \\
\hline & 8. & Confianza del estudiante en el docente & $2,35 \%$ \\
\hline & 9. & Repitencia & $-20,00 \%$ \\
\hline & 10. & Asistencia a clases & $5,88 \%$ \\
\hline & 11. & Expectativas de los padres y apoderados & $10,59 \%$ \\
\hline & 12. & Participación de los padres y apoderados & $1,18 \%$ \\
\hline \multirow[t]{8}{*}{ Establecimiento } & 13. & Número de los profesores & $1,18 \%$ \\
\hline & 14. & $\begin{array}{l}\text { Docentes bien evaluados en Educación } \\
\text { Matemática }\end{array}$ & $3,53 \%$ \\
\hline & 15. & Cobertura curricular en Educación Matemática & $4,71 \%$ \\
\hline & 16. & Conductas relacionadas con el bullying & $-3,53 \%$ \\
\hline & 17. & Nivel socioeconómico & $5,88 \%$ \\
\hline & 18. & $\begin{array}{l}\text { Conocimiento de la gestión directiva y } \\
\text { pedagógica del establecimiento }\end{array}$ & $7,06 \%$ \\
\hline & 19. & Disciplina del establecimiento & $3,53 \%$ \\
\hline & 20. & Satisfacción de los padres y apoderados. & $2,35 \%$ \\
\hline
\end{tabular}

Fuente: Vega y Galaz, 2015. 
Precisamente, por esta multiplicidad de factores, se considera necesario atender a la gran cantidad de relaciones entre los actores que se encuentran en la comunidad escolar, comunidad que corre el riesgo de desarticularse si uno de los factores no comparte sentidos comunes. En el texto, "Gestión de la Buena Convivencia" (MINEDUC, 2013) se entiende el ambiente escolar como:

"contexto o ambiente en el que se producen las interrelaciones, la enseñanza y los aprendizajes en el espacio escolar; está determinado por una serie de condiciones necesarias para la apropiación de los conocimientos, habilidades y actitudes establecidas en el currículum y posibilita -o dificulta- el aprendizaje, no solo de cómo convivir con otros y otras, sino, también, de los contenidos y objetivos de aprendizaje de las diversas asignaturas" (p. 8).

Dando cuenta de la visión específica, sobre la relación ideal que se debe dar en el conjunto de cada comunidad educativa al declarar que: "Mientras más organizado sea el entorno, con mayor claridad, aceptación y consistencia en las normas, con docentes (y adultos en general) que cultivan altas expectativas respecto de sus estudiantes, con modos de relacionarse colaborativos y respetuosos, entre otros factores, más favorable será el ambiente para enseñar y para aprender" (p. 8).

El ideario de esta intención declarada es el documento denominado "Manual de Convivencia Escolar", cuya elaboración en cada escuela o colegio es obligatorio, según lo determinado en el art. 46, letra f de la Ley General de Educación ${ }^{4}$, para regular las relaciones entre los actores educativos al interior del establecimiento educacional. Este reglamento debe establecer claramente "el justo procedimiento en el caso en que se contemplen sanciones, las que en ningún caso podrá contravenir la normativa vigente" (MINEDUC, 2011, p. 5).

\footnotetext{
4 "Contar con un reglamento interno que regule las relaciones entre el establecimiento $\mathrm{y}$ los distintos actores de la comunidad escolar, y que garantice el justo procedimiento en el caso en que se contemplen sanciones. Este reglamento no podrá contravenir la normativa vigente." (Ley 20370 del MINEDUC)
} 


\subsection{La anotación negativa como regulador}

Uno de los instrumentos que se maneja para registrar la acción al interior de establecimiento escolar es el Libro de Clases, cuya función es la de llevar el registro de los acontecimientos más importantes de la relación escolar. En primer lugar, registro de la identificación y organización de cada comunidad curso (incluyendo nombre y direcciones de cada apoderado, indicando la función en la organización del microcentro que constituyen); registro de las asistencias a clases de cada alumno (diario y por hora, incluyendo eventos extraordinarios como el ingreso con retraso a clases, la ausencia entre horas o retiros anticipados); registro de las calificaciones obtenidas por los alumnos; registro de las actividades docentes desarrolladas y registro de las acciones extraordinarias desarrolladas por los alumnos, tanto en forma individual como colectiva, conocido como registro de anotaciones (MINEDUC, 2014).

En la sección de Registro de Anotaciones es en donde se anotan, de forma descriptiva, las acciones desarrolladas por los y las estudiantes que escapan a la norma. Estas acciones deben ser identificadas como negativas o positivas, ya sea mediante una marca visible (+ para positivo y - para negativo), o mediante su palabra explícita (negativa o positiva). La facultad de hacer registros en el libro de clases, en la sección de anotaciones, es exclusiva de los docentes de aula, docentes directivos $\mathrm{u}$ otros profesionales intervinientes en el proceso educativo (inspectores, asistentes de aula, profesionales de apoyo). Dado su carácter oficial, su registro debe tener correlación, en su contenido, con lo estipulado en el Reglamento de Convivencia Escolar, e idealmente, debe corresponderse con los elementos citados como positivos o negativos dentro del mismo reglamento ${ }^{5}$.

${ }^{5}$ En un estudio documental desarrollado en la Región de los Ríos, de verificación de los Reglamentos de Convivencia Escolar de 11 establecimientos educacionales de enseñanza media y básica de segundo ciclo, se constató que solo 1 hace referencia a la anotación positiva como medio a promover, dedicando la mayoría de la reglamentación a indicar el procedimiento para las anotaciones negativas. (Vega, 2017) 
La acumulación de actitudes positivas o negativas puede tener consecuencias directas sobre la permanencia, tanto en su modo como extensión de los y las estudiantes en el establecimiento, aunque no puede incidir por sí mismo, en la suspensión indefinida de matrícula (SuperEduc, 2016).

Según el estudio ya citado de Alarcón (2015), la mayoría de las anotaciones responde a una valoración negativa (considerando actitud, compromiso y gradación), pudiendo determinarse, en el primer indicador, tres componentes del subsistema de actitud: Juicio, afecto y apreciación, cuya preeminencia es mayor sobre la estructura de construcción de las anotaciones. La totalidad de indicadores de valoración son construidos siguiendo el modelo de la Teoría de la Valoración (Ledema, Feez, Y. y White, 1994; White, 2002; Navarro, 2013), profundizado por varios autores en los últimos años (Kaplan, 2004). Los otros subsistemas interactúan con los diversos indicadores, dando cuenta de la complejidad de la percepción directa del sentido del registro, dado que su contexto, muchas veces, es desconocido (figura 2). Al ser una prerrogativa exclusiva de un segmento docente, su utilización es, muchas veces, limitada a ciertas valoraciones.

Figura 2. Diversidad de los sistemas y subsistemas de valoración

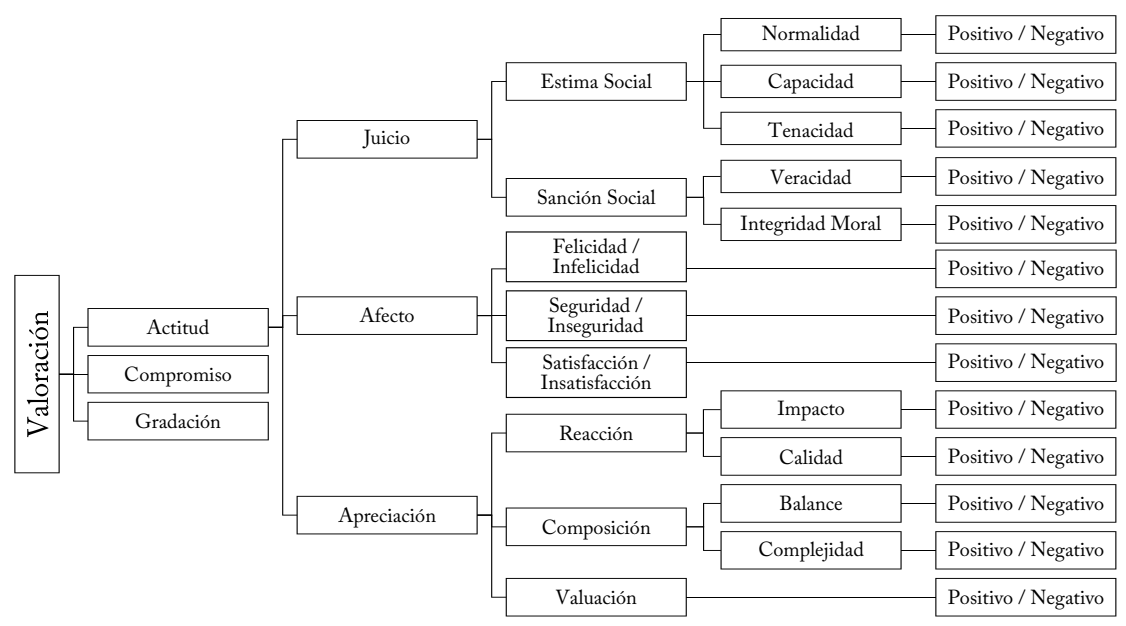

Fuente: Elaboración propia, basada en White, 2002; Navarro, 2003; Kaplan, 2004; Alarcón, 2015. 


\subsection{La anotación negativa desde la propuesta de Austin}

El uso de la anotación negativa en el contexto escolar se transforma en una forma de constatación de la interacción de la comunidad, al interior del establecimiento, cuyo registro da cuenta de una situación de alerta inmediata sobre lo positivo/negativo que una conducta puede conllevar. La constatación realizada por un profesor se transforma, asimismo, en una forma de discurso, que puede ser analizada desde su condición de crítica o juicio negativo (Navarro, 2013). Esta constatación tiene validez en cuanto el docente, por su sola autoridad, es un sujeto creíble, y por lo tanto, garantía de veracidad, confiriéndosele calidad de juez en el micro acto del juicio de acción.

Esto sugiere que la anotación registrada en el libro de clases cumple una función de constatación (Austin, 1982), de modo que aquello que ahí se escribe es lo que es, sin lecturas secundarias. Dado este carácter declarativo, se re-construye la realidad en cuanto a la falta cometida por el o la estudiante, a partir de lo allí registrado, con intención de hacer un seguimiento posterior. En los reglamentos de convivencia escolar, se indica cuáles son las actitudes deseables, en torno a las que se debe desarrollar la convivencia, declaración tanto en términos de actitudes deseadas (perfil del o la estudiante) como de acciones reprochables (faltas estratificadas según nivel). Estas indicaciones, a su vez, tienen directa relación con la legislación vigente, de modo que se desarrolla un sistema de especificación cada vez mayor, en atención de la cultura local respecto de lo deseable, esperable o tolerable en cada comunidad escolar. La anotación negativa surge, de esta forma, como un mecanismo de constatación de la conducta con un doble sentido, a la vez que se erige como una herramienta de control: Herramienta de control inmediato (sanción en el acto de la conducta negativa con fines de corrección inmediata) y herramienta de control mediato (constatación de acto contrario a las normas deseables, en apoyo a alguna acción secundaria). Por lo tanto, la acción de registrar en un libro de clases una anotación negativa responde tanto a micro como a macrosistemas relacionados.

En cuanto a macrosistema, la acción declarativa (acto de constatación) responde a la legislación vigente (MINEDUC, 2015; MINEDUC, 
2009; MINEDUC, 2011b), a los fines de la educación (MINEDUC, 2009), al currículum escolar (MINEDUC, 2013b), al perfil de egreso, a la cultura local, a las conductas deseables según el Manual de Convivencia Escolar (MINEDUC, 2016) y a las relaciones permitidas al interior del aula (MINEDUC, 2004). En cuanto a microsistema, responde a la interacción permitida al interior del aula (norma social), a la relación que se tenga con el docente (relación posible), al conocimiento que tenga el docente del Manual de Convivencia Escolar, a la valoración de la acción observada (escala de valores privada) y a la percepción del alcance del acto de anotar en el libro de clases. Es pues, una declaración con consecuencias relacionadas de forma compleja, en donde el docente tiene la decisión de hacerlo efectivo o no.

Figura 3. Macro y Microsistema de relaciones vinculados en torno al registro de una anotación negativa.

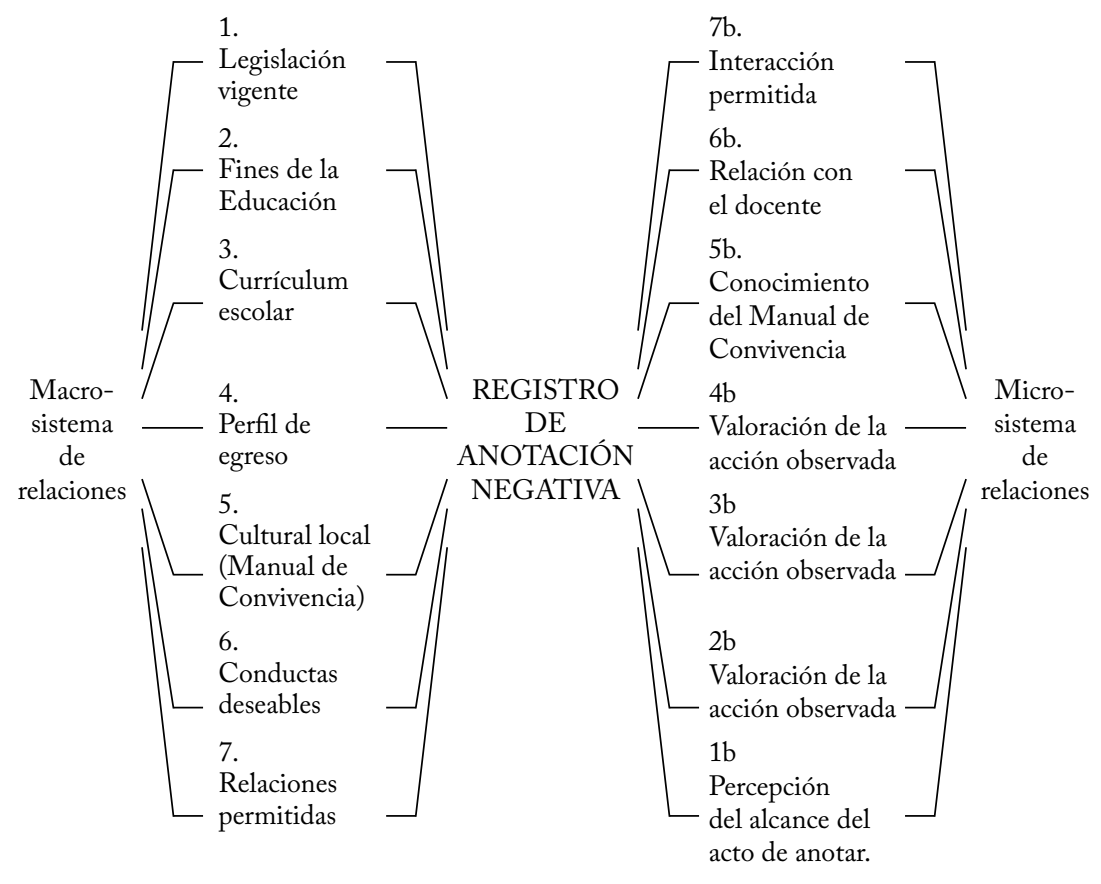

Fuente: Autor, basado en MINEDUC (2004; 2009; 2011; 2011b; 2013b; 2016) 


\subsection{El sentido de la anotación negativa}

Esto significa que cualquier anotación registrada en el Libro de Clases depende de una examinación de todos los elementos posibles de validación mencionados. Esto no es una particularidad de nuestro actual sistema escolar, puesto que ya en las primeras propuestas de organización escolar se contempla la verificación del contenido de la anotación por parte de un censor (Foucault, 2002) ${ }^{6}$, por lo que los límites de la anotación negativa en su constatación están en la verificación del nivel Macro como verificador general, lo que puede incluso sobreponerse al nivel Micro. Así una anotación cuyo contenido sea "Abandona dando un portazo al salir" (Alarcón, 2015, p. 283) tendrá validez de negativa solo si en alguno de los ámbitos de relación del nivel Macro prohíbe la acción indicada. Si no hay prohibición, y si el sujeto agente de la anotación no llega al nivel de defender o clarificar su registro, éste pierde validez, arrastrando tras de sí la validez general del tipo de registro que realice cada sujeto.

Cuando el nivel Macro carece de claridad en su construcción, por ejemplo, en el referente de "cultura local (Manual de Convivencia)", el sistema de validez tambalea, por trasladarse todo el peso de la afirmación al referente del Microsistema "Conocimiento del Manual de Convivencia”, dado que el conocimiento es indicador de capacidad para juzgar de acuerdo a lo declarado, siempre y cuando la declaración sea suficiente y clara. Si no lo es, el "conocimiento del Manual de Convivencia " sólo servirá para dar cuenta de la ambigüedad general del sistema de relaciones posibles, y así una anotación del tipo "Alumna irreverente, durante toda la hora de clases se depila y se maquilla frente a todo el curso" (1,1 R15-10a , Vega, 2017) podrá ser, solamente aceptada como válida, si el Manual de Convivencia establece que maquillarse es una conducta reprochable.

${ }^{6}$ En el texto menciona a propósito de la Escuela para Aprendices de los Gobelinos "en un libro general que llevan los profesores y sus ayudantes debe registrarse cotidianamente la conducta de los alumnos y todo cuanto ocurre en la escuela. Dicho libro se somete periódicamente al examen de un inspector". (Foucault, 2002, p. 45) 
Esto indica que la función de la anotación negativa no podría ser solamente una función de constatación (como hemos indicado más arriba), ya que su cualidad no radica exclusivamente en la declaración (aceptada sin cuestionamiento) del dato declarado. La afirmación no es, por tanto, solo un reflejo de lo ocurrido, sino que es, con mayor posibilidad, un acto de realización (Austin, 1982), que da cuenta de la situación, pero que es sometida todavía a una verificación, lo que destruye la condición de posibilidad de ser un dictamen suficiente en sí mismo.

Entonces, si la anotación negativa es siempre cuestionable, con dependencia del Macrosistema de relaciones, la posibilidad que el $\mathrm{Mi}^{-}$ crosistema de relaciones se haga cargo de la verificación del dato es nula, por cuanto, solamente serviría para justificar la acción declarada por parte del docente, sin que la connotación de negativa sea posible en cuanto que el verificador será la objetivación del Macrosistema. Esto explica de forma clara por qué el texto de Alarcón, al que hemos hecho referencia, toma una perspectiva de análisis desde los sistemas y subsistemas de valoración, y no desde la objetivación del ejercicio del poder, dado que el poder, en este caso, no radica en el sujeto que desarrolla la acción en sí, sino que queda sometido a la concordancia de la acción con el Macrosistema (cuya comprensión de lo bueno deseable ya está objetivada en diversos cuerpos), perdiendo la validez en sí. Por lo mismo, cuando un docente expresa la advertencia "te voy a anotar", con tono amenazante, ejecuta un acto perlocutivo de poco alcance, por cuanto el anotar en sí no tiene validez suficiente, lo que nos abre las perspectivas de análisis hacia una pregunta final ¿qué utilidad puede tener la anotación negativa en el contexto escolar?, o, de forma más directa, ¿tiene utilidad fuera de ser un elemento de control de la conducta en el Macro y Microsistema de relaciones?

\section{Rol de la anotación negativa como recurso de control}

\subsection{El uso de la anotación negativa}

Dada la limitación a que se refiere el uso de la anotación negativa como 
recurso absoluto (por su cuestionable carácter de constatación), nos abre a la pregunta sobre el uso final que puede darle un profesor. $Y$ este puede ser leído desde la lógica del poder, en donde la anotación negativa es una estrategia para la mantención del poder (Dreyfus Yy Rabinow, 2001), en el primer sentido que lo plantea Foucault "medios para alcanzar cierto fin” (p. 257) 7 . Una estrategia permite alcanzar un fin, en el caso de la anotación negativa, mantener la posición de responsabilidad y dominio del aula por lo tanto, el mensaje "te voy a anotar" y el acto de anotar (previos a la anotación en sí), siguen siendo prerrogativa de un solo agente que cuestiona y analiza la dimensión de la acción desarrollada con total dominio de la acción en cuanto a sanción.

Este sistema se respalda en la naturaleza positiva de la relación formativa que se establece en un aula. Nada de lo desarrollado en el aula puede escapar de los sistemas de control a que responden todos los sujetos. Son finalmente sistemas vinculados (la estructura del $\mathrm{Ma}-$ crosistema de relaciones). Así, cada una de las esferas que compone el Macrosistema exige de medios de supervisión para mantener su validez, y así puede ejercer un valor absoluto de uno sobre otro. Por lo tanto, una anotación del tipo "Alumno se intercambia prueba con compañero" (1,1 R41-6, Vega, 2017), sirve para mantener el poder en cuanto la conducta puede ser una práctica social tolerada (Guillamon Yy Acale, 2014), pero cuyas consecuencias pueden ser ponderadas como negativas (por ejemplo, para la valoración de la evaluación, o para la objetividad de la misma como instrumento de verificación de aprendizajes), pero aún, si el docente no hubiera considerado la esfera de la Norma Social como censor para la sanción, todavía podría sancionar en virtud del Manual de Convivencia, y si este no lo contempla

\footnotetext{
7 "La palabra estrategia se emplea actualmente de tres maneras. En primer lugar, para designar los medios empleados para alcanzar cierto fin; es una cuestión de racionalidad que funciona para llegar a un objetivo. En segundo lugar, para designar la manera en que actúa una de las partes en un cierto juego en vistas a lo que piensa que podría ser la acción de los otros y lo que él considera que los otros piensan de la suya; es la manera en que uno busca tener la ventaja sobre los otros. En tercer lugar, para designar los procedimientos usados en una situación de confrontación para privar al oponente de sus medios de combate y reducirlo a abandonar la lucha; por consiguiente, se trata de los medios destinados a obtener una Victoria” (Dreyfus y Rabinow, 2001, p. 257)
} 
tenemos aún (en ascenso) los Planes y Programas de Estudio, el Marco Curricular Nacional, la Ley General de Educación y, finalmente, de forma superior y absoluta, la Legislación Vigente que regula las relaciones entre todos los individuos de un mismo conjunto social (país o nación).

Figura 4. Sistema Cuerpos legales relacionados en el nivel Macrosocial

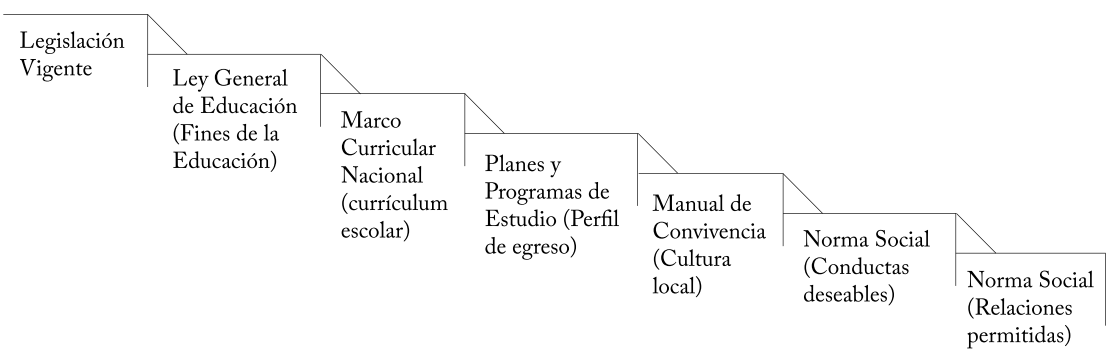

Fuente: Autor, basado en MINEDUC (2004; 2009; 2011; 2011b; 2013b; 2016)

Así, el profesor funciona como un administrador de estrategias de control, dentro de un dispositivo de control mayor como es la sociedad. Sociedad en que la Educación permite/provoca la cohesión social (entendida como ethos societal), pero también la mantención de estructuras globales de control y dominación para el ajuste de la conducta general del individuo en pos de la conducta deseada (Dreyfus y Rabinow, 2001, p. 259). En este sentido, no se debe olvidar que todo ejercicio del poder en forma de relación (relación de poder) provoca resistencias por los sujetos que quedan en situación desmejorada. Como menciona Foucault, no existen relaciones de poder sin resistencias, y estas "son más reales y más eficaces cuando se forman allí mismo, donde se ejercen las relaciones de poder" (Foucault, 1979, p. 171), de ahí que el profesor en su ejercicio, al mismo tiempo motiva y provoca, de forma inevitable, la estrategia de resistencia que puede ser la acción no deseada o la acción deseada, pero no asumida.

Finalmente, queda en la anotación negativa, la alternativa de ser una estrategia de modelamiento en uso, junto con otras posibles estrategias, cuya regulación en el Macrosistema han perdido validez (Torrecilla, 1998; Toro, 2008; Suárez, 2004; Valle-Barbosa, Vega-López, 
Flores-Villavicencio, Yy Muñoz-De la Torre, 2014; Becerra, 2011), principalmente porque la esfera mayor (la legislación vigente), ha regulado en un orden que ha modificado todos y cada uno de los niveles relativos. De hecho, esto es lo particularmente interesante de la sociedad como sistema de control, ya que se mueve con plasticidad, aumentando los márgenes en los que logra mantener la dominación sobre el conjunto, acogiendo la resistencia y dando respuesta a la misma para aumentar los márgenes de lo permitido, pero estableciendo, a su vez, los nuevos límites dentro de los cuales es posible ejercer la nueva dominación, dominación a la cual se deben ajustar todos los sistemas por la necesidad recursiva de mantener un dispositivo de control societal.

Esto se hace notorio en la antigua disposición disciplinaria, que permitía expulsar a un alumno por acumulación de anotaciones negativas. En un sistema tradicional, considerando la escuela como el modelo surgido alrededor de 1.800 en torno a las fábricas, y a su versión humanista-cristiana, promovido, entre otras congregaciones, por los Hermanos de las Escuelas Cristianas ${ }^{8}$ (Foucault, 2002), el uso y recurso del castigo físico era considerado como la norma, dado que la escasa consideración sobre el cuerpo, y la convicción de que su castigo modelaba y moderaba el alma, por lo que era visto como un instrumento para la templanza del ser humano (Valle-Barbosa, Vega-López, Flores-Villavicencio y Muñoz-De la Torre, 2014). Esto se mantuvo, con algunas variantes, hasta que a inicios del S.XX se inicia un proceso de resistencia cada vez mayor, cuyas bases las encontramos ya en Locke, en su tratado "Algunos Pensamientos sobre Educación" (Locke, 1986) 9 que hace que cambie la consideración sobre el castigo físico y se migre hacia la simbolización del castigo, como es la exclusión (visibilizada a través del recurso de la expulsión del centro escolar).

${ }^{8}$ Los Hermanos de los Escuelas Cristianas son conocidos actualmente como "De la Salle", por su fundador, San Juan Bautista De la Salle (1651-1719).

9 "El castigo debe ser empleado solamente en este terreno, el de la formación de las costumbres, y únicamente cuando no se haya logrado convencer al pupilo razonado. En cuanto a la instrucción, se debe propiciar la inclinación hacia el estudio sin recurrir a la vara" (Locke, 1986, p. 17). 
El mecanismo de la expulsión se basa en el sistema de registro de conducta ya referido (Foucault, 2002), en donde, la conducta visibilizada como negativa, y en orden a su reiteración o gravedad, puede apoyar la expulsión (Ministerio de Educación, 2003). Esta fue la práctica utilizada, hasta antes de la entrada en vigencia de la Ley de Inclusión (2015), que permitía como sanción, relativa a la acumulación de faltas de mayor consideración, la expulsión del causante de la acción antes del término del año escolar (Foro Chileno por el Derecho a la Educación, 2015), cuestión que estaba estipulada en los diversos Reglamentos Escolares. Esto, por tanto, se modifica al cambiar el cuerpo legal superior, ya que la Ley de Inclusión (2015) obliga a buscar otras medidas, y en ningún caso se permite la expulsión como acción inmediata (sin relación a la gravedad del hecho), sino como corolario, de un año a otro, de las acciones acumuladas, demostrando que se desarrollaron todas las acciones reparativas y formativas previas (MINEDUC, 2015). Así, la acción de declarar con una anotación negativa la conducta indeseada del alumno, pasa a ser un acto de mayor peso simbólico, pero no de mayor eficacia, al no tener el profesor total autonomía sobre la acción que desarrolla, sino estar sometido al margen que la Ley le confiere. El discurso escrito pierde su eficacia de tal, al requerir otro verificador que puede cuestionar, limitar e incluso anular el contenido declarado, haciendo del profesor un administrador de los mecanismos de control, en un dispositivo de control del cual forma parte (Foucault, 2002).

\section{Conclusiones}

El análisis de las anotaciones negativas, tanto desde su uso (registros escolares), como desde su posibilidad de uso (Micro y Macrosistemas relacionados), dan cuenta de las dificultades de la anotación negativa como un acto directo de declaración absoluta de la acción de un estudiante, particularmente por el hecho de ser sometido, constantemente, a revisión de la condición de veracidad de la misma anotación. Desde este punto de vista, la anotación negativa muchas veces da más cuenta del sentir interno del docente que genera el registro (dentro del Microsistema de relaciones posibles) que de la posibilidad real de ser 
validado como discurso definitivo.

Este elemento agrega una complejidad al ejercicio docente, ya que la anotación negativa puede terminar representando dos posibilidades: o la expresión de un sentir interno (con plena conciencia de su nulo efecto inmediato), o la expresión de una valoración externa con claridad sobre el efecto que pudiera tener, aun cuando su contenido sea valorado en un acto segundo. La sola expresión de un sentir interno, como un acto performativo de sublimación de la frustración por la relación social fallida entre el docente y la conducta del estudiante, abre las posibilidades de análisis de la anotación negativa desde nuevas perspectivas de estudio (el registro como objeto de expresión del locus interno sin relación con el efecto real en el entorno). La segunda posibilidad, la de la valoración externa de una situación, como juicio apreciativo, nos coloca en la perspectiva que este instrumento tendría su valoración preeminente como instrumento de control, administrado por un grupo, cuyo control real sobre el grupo social al que está vinculado, ha perdido eficacia. De hecho, el que la valoración final de la anotación negativa (más allá de la calidad de su construcción) esté situado fuera del individuo que la emite, confirma el dato que la autoridad en sí de la anotación no corresponde al autor, sino al cuerpo legal en que se circunscribe y que, a su vez, pertenece a sistemas cada vez mayores de referencia, como extensión inconsciente de un dispositivo de control como es el cuerpo legal, y finalmente obedece a la concepción de persona que tiene una sociedad en particular.

En el caso particular de Chile (aun cuando la utilización de anotaciones negativas no sea exclusiva de este país), da cuenta de la baja consideración que con el tiempo ha ido adquiriendo el profesor como autoridad en su entorno, al mismo tiempo que da cuenta que las formas de control prevalecen con independencia de la conciencia real que tengan los sujetos que emiten sus discursos. 


\section{Referencias}

Alarcón, M. (2015). Patrones valorativos de actitud en anotaciones en el Libro de Clases: Afectos y apreciaciones de los docentes en un contexto educativo. Literatura y Lingüistica, 31, 269-294.

Ascorra, P. y Crespo, N. (2004). La incidencia del rol docente en el desarrollo del conocimiento metacomprensivo. Psicoperspectivas, III, 23-32.

Austin, J. (1982). Cómo hacer cosas con las palabras. Barcelona: Paidós.

Becerra, J. L. (2011). Del varillazo a la anotación negativa: Transformaciones en el uso de la violencia física como método disciplinario en los colegios fiscalesmunicipales. 1970-2010. Santiago de Chile: Seminario para optar al grado de Licenciado en Historia.

Bravo, D. (2008). La relación entre la evaluación docente y el rendimiento de los alumnos. Santiago de Chile: Centro de Microdatos de la Universidad de Chile.

Brunner, J., Y. y Elaqua, G. (2004). Factores que inciden en una educación efectiva. Evidencia internacional. Recuperado de: http://www.educoea. org/portal/bdigital/lae-ducacion/139/pdfs/139pdf1.pdf

Cerón, F., Y. y Lara, M. (2011). Factores asociados con el rendimiento escolar. Santiago de Chile: Ministerio de Educación.

Cornejo, R., Y.y Redondo, J. (2007). Variables y factores asociados al aprendizaje escolar: una discusión desde la investigación actual. Revista Estudios Pedagógicos, 2, 155-175.

Dreyfus, H., Y. y Rabinow, P. (2001). Michel Foucault, más allá del estructuralismo y la hermenéutica. Buenos Aires: Nueva Visión.

Foro Chileno por el Derecho a la Educación. (2015). Análisis crítico de la Ley que regula la admisión de los y las estudiantes, elimina el financiamiento compartido y prohibe el lucro en establecimientos educacionales que reciben aportes del Estado. Recuperado de: http://www.opech.cl/comunicaciones/2015/05/index_19_05_2015_analisis_ley_inclusion.pdf

Foucault, M. (1979). Microfísica del Poder. Madrid: Las Ediciones de la Piqueta.

Foucault, M.(2002). Vigilary Castigar. Nacimiento de la Prisión. Buenos Aires: Siglo XXI.

Guillamon, J. Y. y Acale, M. (2014). Evaluación y calificación en el ECTS: análisis de casos y conflictos. XVI Encuentro Estatal de Defensores Universitarios. Recuperado: http://www.upv.es/entidades/DCU/info/U0675603.pdf 
Ledema, R., Feez, S., Y. y White, P. (1994). Media Literacy. Sydney: Disadvantaged Schools Program. NSW: Department of School Education.

Irene, M., Y. y Bareño, T. (2015). Variables asociadas al desempeño escolar. Bogotá: Editorial Milla.

Kaplan, N. (2004). Nuevos desarrollos en el estudio de la evaluación en el Lenguaje: La Teoría de la Valoración. Boletín de Lingüistica, 22, 52-78.

Locke, J. (1986). Pensamientos sobre la Educación. Madrid: Ediciones Akal.

Marchesi, Á., Lucena, R., Y. y Ferrer, R. (2006). La opinión de los alumnos sobre la calidad de la Educación. Madrid: Fundación Hogar del Empleado. MINEDUC (2003). Educación: Derecho y responsabilidad de todos. Folleto $\mathrm{n}^{\circ}$ 7. Expulsión y cancelación de matriculas. Santiago de Chile: Ministerio de Educación de Chile.

MINEDUC (2004). Ley 19979, Modifica el régimen de Jornada Escolar Completa Diurna y otros cuerpos legales. Santiago de Chile: Ministerio de Educación, 28 de Octubre de 2004.

MINEDUC (2009). Ley 20370. Establece la Ley General de Educación. Santiago de Chile: Ministerio de Educación, 17 de Agosto de 2009.

MINEDUC (2011). Orientaciones para elaboración y revisión de reglamentos de convivencia escolar. Santiago de Chile: Ministerio de Educación.

MINEDUC (2011b). Ley 20536, Sobre Violencia Escolar. Santiago de Chile: Ministerio de Educación, 08 de Septiembre de 2011.

MINEDUC (2013). Recursos para enseñar y aprender. Recuperado: http:// www.curriculumenlineamineduc.c1/605/w3-channel.html

MINEDUC (2013b). Gestión de la Buena Convivencia. Santiago de Chile: Ministerio de Educación.

MINEDUC (2014). Circular N 1, Establecimientos Educacionales Subvencionados Municipales y Particulares. Santiago de Chile: Ministerio de Educación.

MINEDUC (2015). Ley 20845. Biblioteca del Congreso Nacional de Chile Santiago de Chile: Gobierno de Chile, 29 de mayo de 2015. Recuperado: https://www.leychile.c1/Navegar?idNorma=1078172.

MINEDUC (2016). Orientaciones para la revisión de los Manuales de Convivencia Escolar. Actualización según la Ley de Inclusión. Santiago de Chile: Ministerio de Educación.

Navarro, F. (2013). Estrategias y recursos de evaluación negativa: aportes para un modelo de análisis sistémico-discursivo. Boletín de Filología, Tomo XLVIII, 69-96. 
Suárez, M. (2004). Los castigos y otras estrategias disciplinares vistos a través de los recuerdos escolares. Revista de Educación, 335, 429-443.

SuperEduc, S. d. (2016). Denuncias Superintendencia de Educación. Obtenido de Medidas disciplinarias, expresadas en tres categorías. Recuperado: $\mathrm{http}: / /$ denuncias.supereduc.cl/cuestionario1/denuncias_tematicos.html

Toro, P. (2008). Disciplina y castigos: fragmentos de la cultura escolar en los liceos de hombres en Chile en la segunda mitad del Siglo XIX. Cuadernos Interculturales, 6(11), 127-144.

Torrecilla, L. (1998). Niñez y castigo, historia del castigo escolar. Valladolid: Universidad de Valladolid.

Valle-Barbosa, M., Vega-López, M., Flores-Villavicencio, M., Y Muñoz-De la Torre, A. (2014). Los castigos escolares utilizados como técnica para mantener la disciplina en las escuelas mexicanas a partir del Siglo XIX. Revista Educación y Desarrollo, 28, 61-68.

Vega, J. (2017). Base de datos de anotaciones negativas, primer año medio 2013. Valdivia.

Vega, J. (2017). Estudio descriptivo del uso de anotaciones en la Región de Los Ríos. Valdivia, Chile.

Vega, J., YY Galaz, A. (2015). Evidencias para una transformación y complejización del modelo chileno de evaluación docente. Educacao e Pesquisa, 41 (1), 171-183.

Vygotsky, L. (2009). El desarrollo de los procesos psicológicos superiores. Barcelona: Crítica.

White, P. (2002). Appraisal. En J. Verschueren, J.-O. Östman, J. Blommaert, Y C. Bulcan, The handbook of pragmatics, vol. 8. Amsterdam: John Benjamins Publishing Company. 